\title{
DEFICIÊNCIA DE MACRONUTRIENTES EM MUDAS DE SANGRA D’ÁGUA (Croton urucurana, Baill.)
}

\author{
Maria Claudia Mendes Sorreano ${ }^{1}$, Eurípedes Malavolta ${ }^{2}$, Denis Herisson da Silva ${ }^{3}$, \\ Cleusa Pereira $\mathrm{Cabral}^{4}$, Ricardo Ribeiro Rodrigues ${ }^{5}$
}

(recebido: 20 de outubro de 2009; aceito: 28 de abril de 2011)

RESUMO: Com o presente trabalho, objetivou-se descrever os sintomas de deficiência de nitrogênio (N), fósforo (P), potássio (K), cálcio $(\mathrm{Ca})$, magnésio $(\mathrm{Mg})$, enxofre $(\mathrm{S})$ e avaliar o efeito da omissão desses nutrientes na composição mineral das folhas de mudas de sangra d'água (Croton urucurana). O experimento foi desenvolvido em casa de vegetação, em blocos ao acaso, com três repetições e sete tratamentos, empregando-se a técnica da diagnose por subtração. Foram observados os sintomas das deficiências e medidos os parâmetros biométricos, a produção de matéria seca e determinados os teores foliares. A omissão dos macronutrientes resultou em alterações morfológicas traduzidas em anormalidades visíveis, sendo que, os primeiros sintomas surgiram nos tratamentos com omissão de N, K, Ca e Mg, seguindo-se os tratamentos com omissão de P e S. A omissão de N, Ca e Mg provocou a maior redução no desenvolvimento em altura, no diâmetro do caule e no número de folhas e ramos. A produção de biomassa das mudas mostrou-se pouco influenciada pelos tratamentos, não apresentando diferença significativa na produção de matéria seca.

Palavras-chave: Espécie nativa, diagnose foliar, nutrição mineral de plantas.

\section{DEFICIENCY OF MACRONUTRIENTS IN SANGRA D’ÁGUA (Croton urucurana, Baill.) SEEDLINGS}

\begin{abstract}
This study had the objectives of inducing and describing deficiency symptoms of nitrogen, phosphorus, potassium, calcium, magnesium and sulfur, and evaluating the effect of nutrient omission on the foliar mineral composition of "sangra d'água" (Croton urucurana) young plants. The experiment was carried out in a green house and used the technique of diagnosis by subtraction. The experimental design was of randomized blocks, with seven treatments and three replicates. Macronutrient omission led to morphological alterations expressed by visual symptoms being the first symptons occurring on the treatments with omission of $\mathrm{N}, \mathrm{K}$, Ca and Mg treatments, and later on the treatments with omission of $\mathrm{P}$ and $\mathrm{S}$. Growth parameters, namely height, diameter, number of leaves and of branches were affected by the treatments with omission of N, Ca and Mg. Dry matter yield was not significantly decreased by the omission of macronutrients.
\end{abstract}

Key words: Native specie, foliar nutrient diagnosis, mineral plant nutrition.

\section{INTRODUÇÃO}

A espécie Croton urucurana Baill. (sangra d'água) pertence à família Euphorbiacea; árvore de 7-14 m, com tronco de 25-35 cm de diâmetro com folhas simples, pubescentes, prateadas na face inferior, de $9-18 \mathrm{~cm}$ de comprimento (LORENZI, 1992).

Árvore pioneira, de pequeno a médio porte, heliófita, de crescimento rápido e ciclo de vida curto, abundante em diversas formações florestais brasileiras, especialmente na Floresta Estacional Semidecídual. Tolera encharcamento e inundações, formando maciços quase puros em terrenos instáveis e aluviões às margens dos rios, mas ocorre também em clareiras e bordas de mata em terrenos secos de encosta, sendo resistente a geadas fracas (DURIGAN et al., 2002).

Embora a eficácia e a segurança do uso dessa espécie não tenham sido, ainda, comprovadas cientificamente, sua utilização vem sendo feita pelo povo com base na tradição popular que lhe atribui propriedades antibacteriana, antihemorrágicas, antiinflamatória, antisséptica, antiviral e cicatrizante (LORENZI, 2002). No entanto, a grande importância da mesma está na sua utilização em reflorestamentos com finalidade de recuperação de áreas,

\footnotetext{
${ }^{1}$ Bióloga, Professora Dra. em Ecologia Aplicada - Centro de Energia Nuclear na Agricultura - Universidade de São Paulo/USP - Cx. P. 96 - 13400-970 Piracicaba, SP - mcmsorre@gmail.com

${ }^{2}$ In memoriam

${ }^{3}$ Engenheiro Agrônomo, Doutorando em Ciências - Centro de Energia Nuclear na Agricultura - Universidade de São Paulo/USP - Cx. P. 96 13400-970 -Piracicaba, SP - dhsilva@cena.usp.br

${ }^{4}$ Bióloga, Especialista em Solos e Meio Ambiente - Centro de Energia Nuclear na Agricultura - Universidade de São Paulo/USP - Cx. P. 96 13400-970 -Piracicaba, SP - cabral@cena.usp.br

${ }^{5}$ Biólogo, Professor Dr. em Biologia Vegetal - Escola Superior de Cultura Luiz de Queiroz/ESALQ - Universidade de São Paulo/USP - Cx. P. 9 13418-900 - Piracicaba, SP - rrr@esalq.usp.br
}

Cerne, Lavras, v. 17, n. 3, p. 347-352, jul./set. 2011 
como sombreadora de espécies mais tardias, especialmente na composição de matas ciliares, em solos secos, mesmo em regiões de cerradão (DURIGAN et al., 2002).

Apesar de ser uma espécie de grande utilização tanto na tradição popular como em projetos de recuperação de áreas degradadas, informações sobre as exigências nutricionais do Croton urrucurana são escassas na literatura. Contudo, deficiências minerais e distúrbios de crescimento são comuns em espécies arbóreas com alto potencial de crescimento (SANGINGA et al., 1991).

Sorreano et al. (2008) observaram para a mesma espécie os sintomas de deficiência de boro (B), cobre $(\mathrm{Cu})$, ferro $(\mathrm{Fe})$, manganês $(\mathrm{Mn})$, molibdênio (Mo), zinco (Zn) e o seu efeito na composição mineral das folhas e no crescimento das mudas. A espécie mostrou-se sensível à omissão de $\mathrm{Cu}, \mathrm{Mn}$ e $\mathrm{Mo}$, sendo os primeiros a mostrarem sintomas de deficiência visual aos 40 dias após o inicio dos tratamento, seguidos pelo B, Fe e Mo com deficiências visíveis aos 60 dias após o inicio dos tratamento. Os autores observaram também a redução no desenvolvimento em altura e diâmetro do colo causados principalmente pela omissão de B, Mn e Zn.

Estudos realizados por Sanginga et al. (1991) constataram que a produtividade das espécies arbóreas com alto potencial de crescimento, como, por exemplo, a espécie Croton urucurana, é freqüentemente limitada por restrições nutricionais e hídricas, tornando necessário, para o êxito da implantação dessas espécies em solos marginais, o conhecimento de suas necessidades nutricionais, o que ajudará na escolha de espécies adequadas a ambientes de baixa fertilidade.

Em solos brasileiros, a probabilidade de ocorrer deficiência de um macronutriente é maior que a de micronutrientes (RAIJ, 1991), podendo ainda, ser agravada por erros de adubação ou de manejo do solo. Por exemplo, pode ocorrer a deficiência induzida de nitrogênio $(\mathrm{N})$ em solos com suprimento limitado de fósforo (P) (ARAUJO; MACHADO, 2006), o que é comum, em razão da carência desse elemento na maioria dos solos brasileiros (RAIJ, 1991).

A deficiência de potássio $(\mathrm{K})$, por sua vez, pode ocorrer com a calagem excessiva, ou mesmo, pela inibição competitiva com o magnésio $(\mathrm{Mg})$. A absorção de cálcio (Ca) pode ser comprometida com altas taxas de $\mathrm{K} \mathrm{e} \mathrm{Mg.}$ Entretanto, baixos teores $\mathrm{K}, \mathrm{Ca}$ e $\mathrm{Mg}$ podem prejudicar a absorção de enxofre (S), pois os mesmos, atuam como cátions carregadores, facilitando a sua absorção (MALAVOLTA, 2006).

Cerne, Lavras, v. 17, n. 3, p. 347-352, jul./set. 2011
Tendo em vista a importância dessa espécie e a falta de informações sobre as exigências nutricionais da mesma, com o presente trabalho, objetivou-se induzir e descrever os sintomas da deficiência de nitrogênio, fósforo, potássio, cálcio, magnésio e enxofre, avaliando os efeitos dessas deficiências na composição mineral do tecido foliar e sua interferência no desenvolvimento das plantas.

\section{MATERIAL E MÉTODOS}

O projeto foi realizado em casa de vegetação do Laboratório de Nutrição Mineral de Plantas do Centro de Energia Nuclear na Agricultura, Universidade de São Paulo, Piracicaba-SP.

O delineamento experimental utilizado foi o de blocos ao acaso, com três repetições. As mudas foram transplantadas para vasos com capacidade para 2 litros, onde permaneceram por três semanas em solução de Johnson et al. (1957), modificada, com a metade da concentração da solução original, com todos os macros e micronutrientes. Após esse procedimento, as mudas foram transplantadas para vasos com as soluções correpondentes aos tratamentos, a saber: completo (todos os macro e micronutrientes), omissão de N, omissão de P, omissão de $\mathrm{K}$, omissão de $\mathrm{Ca}$, omissão de $\mathrm{Mg}$, omissão de S. Em todos esses tratamentos, foram fornecidos os micronutrientes.

A solução nutritiva completa apresentou a seguinte composição: $3,0 \mathrm{~mL}$ de $\mathrm{KNO}_{3} 1 \mathrm{~mol} / \mathrm{L} ; 2,0 \mathrm{~mL}$ de $\mathrm{Ca}\left(\mathrm{NO}_{3}\right) \cdot 4 \mathrm{H}_{2} \mathrm{O} 1 \mathrm{~mol} / \mathrm{L} ; 1,0 \mathrm{~mL}$ de $\mathrm{NH}_{4} \mathrm{H}_{2} \mathrm{PO}_{4} 1 \mathrm{~mol} / \mathrm{L}$; $0,5 \mathrm{~mL}$ de $\mathrm{MgSO}_{4} \cdot 7 \mathrm{H}_{2} \mathrm{O} 1 \mathrm{~mol} / \mathrm{L} ; 0,5 \mathrm{~mL}$ de Fe-EDTA $0,2 \mathrm{M} ; 0,5 \mathrm{~mL}$ de $\mathrm{KCl} 0,05 \mathrm{~mol} / \mathrm{L} ; 0,5 \mathrm{~mL}$ de $\mathrm{H}_{3} \mathrm{BO}_{3}$ $0,02 \mathrm{~mol} / \mathrm{L} ; 0,5 \mathrm{~mL}$ de $\mathrm{MnSO}_{4} \cdot \mathrm{H}_{2} \mathrm{O} 0,002 \mathrm{~mol} / \mathrm{L} ; 0,5$ $\mathrm{mL}$ de $\mathrm{ZnSO}_{4} .7 \mathrm{H}_{2} \mathrm{O} 0,002 \mathrm{~mol} / \mathrm{L} ; 0,5 \mathrm{~mL} \mathrm{CuSO}_{4} .5 \mathrm{H}_{2} \mathrm{O}$ $0,0005 \mathrm{~mol} / \mathrm{L} ; 0,5 \mathrm{~mL} \mathrm{H}_{2} \mathrm{MoO}_{4}\left(85 \% \mathrm{MoO}_{3}\right) 0,0005$ $\mathrm{mol} / \mathrm{L}$ por litro. Nas soluções dos demais tratamentos as concentrações foram idênticas as da solução completa, exceto quanto ao nutriente omitido. $\mathrm{O} \mathrm{pH}$ foi mantido em torno de 5,5.

A cada três semanas foram realizadas a troca das soluções nutritivas e os levantamentos das variáveis biométricas (altura total, diâmetro do caule, número de folhas e ramos). As mudas permaneceram na casa de vegetação até apresentarem sintomas visuais de deficiência bem acentuados, quando foram colhidas separando-as em parte aérea (folhas + ramos + caule) e raízes.

As diferentes partes foram lavadas em água deionizada e postas a secar em estufa de circulação forçada, a uma temperatura variando de $65^{\circ} \mathrm{C}$ a $70^{\circ} \mathrm{C}$, até atingir 
massa constante. Após a secagem, foi determinada a massa da matéria seca da parte aérea (folhas + ramos + caule) e da raiz, que em seguida foram moídas em moinho tipo Wiley, para serem analisadas quimicamente. O preparo do extrato e a determinação analítica do material vegetal foram realizados segundo Malavolta et al. (1997).

\section{RESULTADOS E DISCUSSÃO}

\subsection{Sintomas de deficiências}

As plantas em solução com omissão dos nutrientes manifestaram sintomas visuais de deficiência em épocas diferentes. Os primeiros sintomas ocorreram nas plantas com a omissão de N, K, Ca e Mg, aos 30 dias após o início dos tratamentos, seguindo-se as plantas com omissão de $\mathrm{P}$ e S, aos 45 dias (Figura 1). As mudas permaneceram na casa de vegetação por um período de 4 meses.

\subsubsection{Nitrogênio $(\mathrm{N})$}

As plantas deficientes apresentaram-se menores, com folhas amareladas, inicialmente as mais velhas, resultado provável da proteólise e redistribuição; ângulo agudo entre caule e folhas; redução da ramificação decorrente da inibição de gemas axilares, resultando em caules menos ramificados; senescência precoce da folhas. Com a intensificação dos sintomas, aos 60 dias do inicio dos tratamentos, foi observado o desenvolvimento de manchas avermelhadas em todo o limbo, em razão do acúmulo de antocianina, o que é típico de falta de $\mathrm{N}$.

$\mathrm{O}$ desenvolvimento reduzido das mudas pode estar relacionado ao papel desempenhado pelo elemento no metabolismo da planta, já que o nitrogênio é um constituinte de muitos componentes da célula vegetal, incluindo aminoácidos e ácidos nucléicos (TAIZ; ZEIGER, 2004).

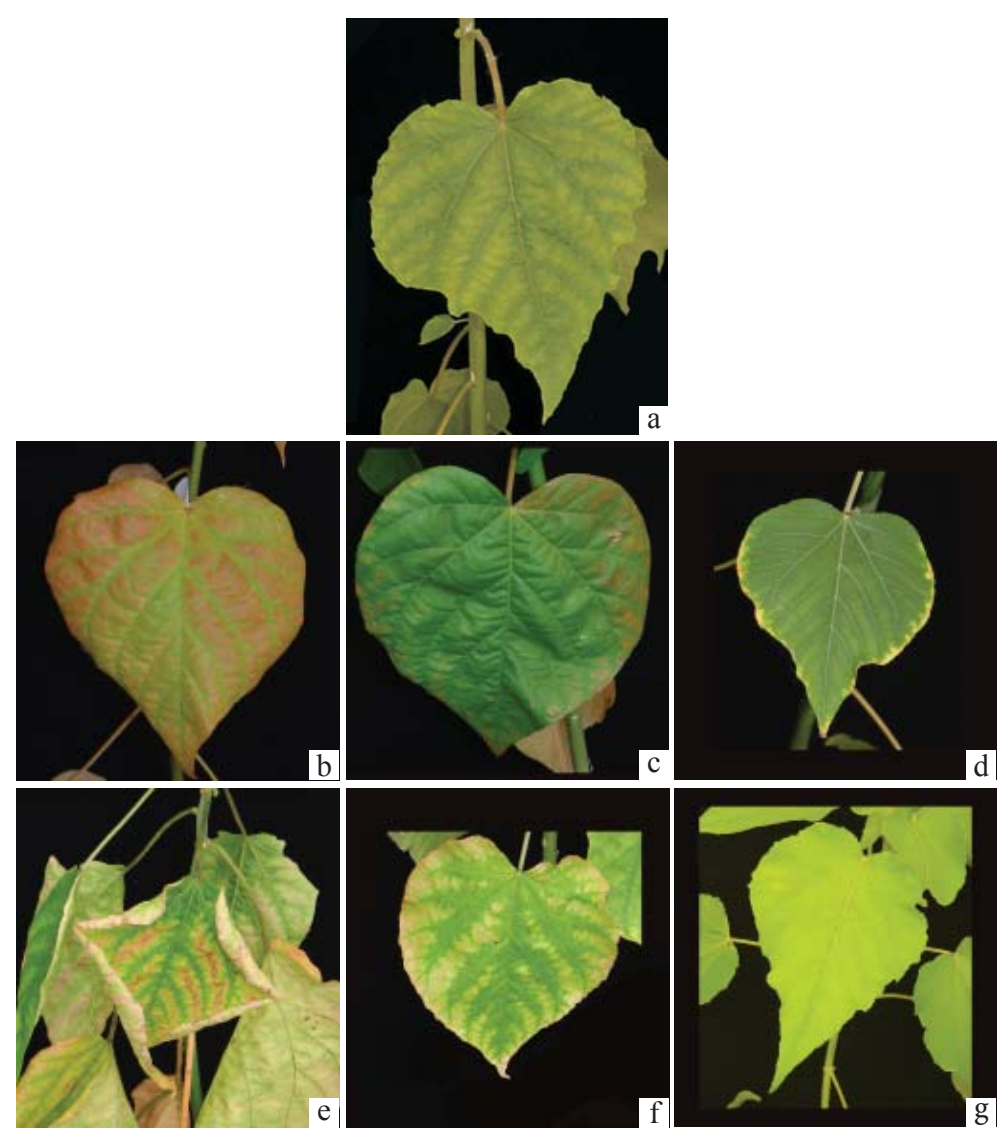

Figura 1 - Sintomatologia das deficiências nutricionais em Croton urucurana, cultivadas em solução nutritiva completa (a), omissão de nitrogênio (b), fósforo (c), potássio (d), cálcio (e), magnésio (f) e enxofre (g).

Figura 1 - Deficiency symptomatology in Croton urucurana seedlings, cultivated in complete nutritive solution (a) or in solutions with omission of nitrogen (b), phosphorus (c), potassium (d), calcium (e), magnesium ( $f$ ) and sulfur $(g)$.

Cerne, Lavras, v. 17, n. 3, p. 347-352, jul./set. 2011 
Os teores foliares de $\mathrm{N}$, encontrados tanto nos tratamentos completos $\left(16,1 \mathrm{~g} \mathrm{~kg}^{-1}\right)$ como sob omissão $\left(6,5 \mathrm{~g} \mathrm{~kg}^{-1}\right)$ foram próximos aos encontrados por Duboc (1994) para Hymenaea courbaril com teores entre $15,2 \mathrm{~g} \mathrm{~kg}^{-1}$ e $7,1 \mathrm{~g} \mathrm{~kg}^{-1}$ e por Viégas et al. (2002) para Annona muricata com teores entre $14,7 \mathrm{~g} \mathrm{~kg}^{-1}$ e $8,8 \mathrm{~g} \mathrm{~kg}^{-1}$.

\subsubsection{Fósforo $(\mathrm{P})$}

As plantas deficientes apresentaram, inicialmente, nas folhas mais velhas, coloração verde-azulada com pouco brilho. Com a intensificação dos sintomas, aos 90 dias do inicio dos tratamentos, foi observado o desenvolvimento de manchas avermelhadas em todo o limbo. Esses sintomas podem estar relacionados ao acúmulo de antocianina, o que é comum também quando há falta de P. As mudas apresentaram redução do crescimento e da ramificação, em razão da inibição de gemas axilares, resultando em caules menos ramificados.

Um efeito primário do $\mathrm{P}$ sobre o crescimento autotrófico é a sua participação na energia química produzida na respiração e na fotossíntese. Como vários processos metabólicos, direta ou indiretamente, dependem desse suprimento energético, a nutrição inadequada de $\mathrm{P}$ pode afetar vários processos, incluindo síntese protéica e de ácidos nucléicos. Assim, plantas com deficiência de P têm o seu crescimento retardado (MENGEL; KIRKBY, 1987).

Os teores foliares de $\mathrm{P}$ encontrados tanto nos tratamentos completos $\left(5,5 \mathrm{~g} \mathrm{~kg}^{-1}\right)$ como sob omissão $\left(0,7 \mathrm{~g} \mathrm{~kg}^{-1}\right)$ foram próximos aos encontrados por Marques et al. (2004) para Schizolobium amazonicum com teores entre $4,3 \mathrm{~g} \mathrm{~kg}^{-1}$ e $0,9 \mathrm{~g} \mathrm{~kg}^{-1}$.

\subsubsection{Potássio $(\mathrm{K})$}

Os primeiros sintomas da deficiência de $\mathrm{K}$ foram constatados em folhas mais velhas na forma de clorose, seguida de necrose das margens e pontas das folhas. Com a intensificação dos sintomas, aos 90 dias do inicio dos tratamentos, foi observada a diminuição da dominância apical.

Sendo o $\mathrm{K}$ ativador de numerosas enzimas, sua deficiência acarreta distúrbios em eventos metabólicos como, por exemplo, acumulação de compostos nitrogenados livres ou solúveis. Esses compostos podem ser aminoácidos, amidas e amônia, além de aminas, produtos da descarboxilação de aminoácidos, como putrescina, $\mathrm{N}$-carbamilputrescina e agmatina. A concentração desses compostos seria responsável pelas manchas necróticas que aparecem em folhas deficientes (EPSTEIN; BLOOM, 2005; MALAVOLTA; CROCOMO, 1982).

Cerne, Lavras, v. 17, n. 3, p. 347-352, jul./set. 2011
Os teores foliares de $\mathrm{K}$ encontrados tanto nos tratamentos completos como sob omissão do nutriente foram respectivamente: $34,1 \mathrm{~g} \mathrm{~kg}^{-1}$ e $3,5 \mathrm{~g} \mathrm{~kg}^{-1}$.

\subsubsection{Cálcio}

As plantas deficientes apresentaram, inicialmente, clorose internerval das folhas mais novas. Com a intensificação dos sintomas, aos 60 dias do inicio dos tratamentos, houve necrose, murchamento e colapso do pecíolo; morte das gemas terminais e gemas laterais dormentes.

$\mathrm{O}$ aparecimento dos sintomas nas áreas marginais das folhas mais jovens e outros tecidos novos devese à falta de remobilização desse nutriente na planta. $\mathrm{O}$ Ca é essencial para manter a integridade estrutural das membranas e das paredes celulares. Quando há deficiência, as membranas começam a "vazar", a compartimentação celular é rompida e a ligação do $\mathrm{Ca}$ com a pectina da parede celular é afetada (MALAVOLTA, 2006), provocando a desestruturação das plantas deficientes em $\mathrm{Ca}$.

Os teores foliares de $\mathrm{Ca}$ encontrados tanto nos tratamentos completos $\left(15,8 \mathrm{~g} \mathrm{~kg}^{-1}\right)$ como sob omissão $\left(2,2 \mathrm{~g} \mathrm{~kg}^{-1}\right)$ foram próximos aos encontrados por Viégas et al. (2002) para Annona muricata com teores entre 14,1 g $\mathrm{kg}^{-1}$ e $3,4 \mathrm{~g} \mathrm{~kg}^{-1}$.

\subsubsection{Magnésio}

A clorose internerval, seguinda de necrose das folhas mais velhas, foram os sintomas mais evidentes da deficiência desse elemento. A despigmentação é característica determinante dos efeitos da deficiência de $\mathrm{Mg}$, pois esse elemento é parte da estrutura da molécula de clorofila e sua deficiência acarreta clorose (TAIZ; ZEIGER, 2004). O Mg é facilmente redistribuído na planta, por isso, os sintomas de deficiência geralmente aparecem primeiro nas folhas mais velhas.

Os teores foliares de $\mathrm{Mg}$ encontrados tanto nos tratamentos completos $\left(8,3 \mathrm{~g} \mathrm{~kg}^{-1}\right)$ como sob omissão $(4,1$ $\mathrm{g} \mathrm{kg}^{-1}$ ) foram próximos aos encontrados por Mendonça et al. (1999) para Myracrodruon urudeuva com teores entre $6,2 \mathrm{~g} \mathrm{~kg}^{-1}$ e $3,4 \mathrm{~g} \mathrm{~kg}^{-1}$.

\subsubsection{Enxofre}

As plantas deficientes apresentaram clorose uniforme das folhas mais novas, as quais mostraram-se pequenas, com enrolamento das margens, seguidas de necrose e queda das mesmas. 
Como o S é um constituinte essencial das proteínas, a deficiência desse elemento resulta na inibição da síntese de proteínas e aminoácidos, contendo esse elemento, como, por exemplo, a metionina e a cisteína (MARSCHNER, 1995).

Os teores foliares de $\mathrm{S}$ encontrados tanto nos tratamentos completos $\left(3,4 \mathrm{~g} \mathrm{~kg}^{-1}\right)$ como sob omissão $\left(1,8 \mathrm{~g} \mathrm{~kg}^{-1}\right)$ foram próximos aos encontrados por Marques et al. (2004) para Schizolobium amazonicum com teores entre $2,7 \mathrm{~g} \mathrm{~kg}^{-1}$ e 2,2 $\mathrm{g} \mathrm{kg}^{-1}$ e por Viégas et al. (2002) para Annona muricata com teores entre $5,3 \mathrm{~g} \mathrm{~kg}^{-1}$ e $2,3 \mathrm{~g} \mathrm{~kg}^{-1}$.

\subsection{Variáveis biométricas e produção de biomassa}

As plantas apresentaram diferenças significativas $(\mathrm{P} \geq 0,05)$ nas variáveis biométricas com omissão de macronutrientes (Tabela 1), sendo que, o N, Ca e $\mathrm{Mg}$ foram os nutrientes que mais influenciarm negativamente o desenvolvimento da espécie.

Tabela 1 - Altura, diâmetro do colo, número de ramos e folhas nos tratamentos com omissão de macronutrientes em Croton urucurana (sangra d'água).

Table 1 - Height, stem diameter, number of branches and leaves in seedlings of Croton urucurana (sangra d'água) submitted to the omission of macronutrient or to complete nutrient solution.

\begin{tabular}{lcccc}
\hline Tratamento & Altura & $\begin{array}{c}\text { Diâmetro } \\
\text { do colo }\end{array}$ & $\begin{array}{c}\mathrm{N}^{\mathrm{o}} \\
\text { Ramos }\end{array}$ & $\begin{array}{c}\mathrm{N}^{\mathrm{o}} \\
\text { Folhas }\end{array}$ \\
\hline Completo & -----cm----- ----mm---- & & \\
Omissão de N & $42,17 \mathrm{a}^{(1)}$ & $10,51 \mathrm{a}$ & $1,00 \mathrm{~b}$ & $16,70 \mathrm{ab}$ \\
Omissão de $\mathrm{P}$ & $66,92 \mathrm{a}$ & $9,23 \mathrm{bc}$ & $1,00 \mathrm{~b}$ & $11,84 \mathrm{~b}$ \\
Omissão de K & $61,67 \mathrm{ab}$ & $9,10 \mathrm{abc}$ & $1,28 \mathrm{ab}$ & $16,64 \mathrm{ab}$ \\
Omissão de Ca & $47,06 \mathrm{bcde}$ & $7,85 \mathrm{bc}$ & $1,2 \mathrm{ab}$ & $19,28 \mathrm{ab}$ \\
Omissão de Mg & $51,03 \mathrm{bcde}$ & $7,91 \mathrm{bc}$ & $1,38 \mathrm{ab}$ & $17,46 \mathrm{ab}$ \\
Omissão de S & $67,29 \mathrm{a}$ & $9,26 \mathrm{ab}$ & $1,47 \mathrm{ab}$ & $16,70 \mathrm{ab}$ \\
\hline CV (\%) & 21,67 & 21,91 & 79,25 & 67,29 \\
Média & 55,24 & 8,51 & 1,59 & 18,50 \\
Valor de $\mathrm{F}^{(2)}$ & $5,52 * * *$ & $1,70 *$ & $2,22 * *$ & $1,97 * *$ \\
\hline
\end{tabular}

${ }^{(1)}$ Médias seguidas pela mesma letra nas colunas não apresentam diferença significativa, a $5 \%$ de probabilidade pelo teste de Tukey. ${ }^{(2) * * *, * *}$, * e NS: significativo ao nível de $1 \%, 5 \%, 10 \%$ de probabilidade e não significativo.

$\mathrm{O}$ crescimento em altura das mudas mostrou-se indiferente à omissão de $\mathrm{P}$ e $\mathrm{S}$ em relação ao tratamento completo. Comportamento semelhante foi observado por
Venturin et al. (2005) mudas de Eremanthus erythropappus (candeia), em que o crescimento em altura não foi influenciada pela omissão de P e S.

A produção de biomassa (Tabela 2) mostrou-se pouco influenciada pelos tratamentos não apresentando diferença significativa, no entanto, esses resultados podem estar associados, em parte, ao pequeno número de repetições e elevado coeficiente de variação no experimento.

Tabela 2 - Produção de matéria seca total (MST), matéria seca da parte aérea (MSPA), matéria seca da do sistema radicular (MSSR) e relação MSSR/MSPA nos tratamentos com omissão de macronutrientes em Croton urucurana (sangra d'água).

Table 2 - Total dry weight (MST), leaves and stems dry weight (MSPA), root dry weight (MSSR) and MSSR/MSPA ratio in seedlings of Croton urucurana (sangra d'água) submitted to the omission of macronutrient or to complete nutrient solution.

\begin{tabular}{|c|c|c|c|c|}
\hline \multirow{2}{*}{ Tratamento } & MS Total & MSPA & MSSR & MSSR/MSPA \\
\hline & \multicolumn{4}{|c|}{----------------------g/planta---------------------- } \\
\hline Completo & $53,27 \mathrm{a}^{(1)}$ & $40,24 a$ & $13,03 \mathrm{a}$ & $0,31 \mathrm{a}$ \\
\hline Omissão de N & $17,63 \mathrm{a}$ & $13,12 \mathrm{a}$ & $4,51 \mathrm{a}$ & $0,34 \mathrm{a}$ \\
\hline Omissão de P & $52,59 \mathrm{a}$ & $40,80 \mathrm{a}$ & $11,71 \mathrm{a}$ & $0,30 \mathrm{a}$ \\
\hline Omissão de K & $40,78 \mathrm{a}$ & $32,39 a$ & $8,39 \mathrm{a}$ & $0,28 \mathrm{a}$ \\
\hline Omissão de $\mathrm{Ca}$ & $31,65 \mathrm{a}$ & $24,95 \mathrm{a}$ & $6,70 \mathrm{a}$ & $0,41 \mathrm{a}$ \\
\hline Omissão de Mg & $33,87 \mathrm{a}$ & $26,05 \mathrm{a}$ & $7,82 \mathrm{a}$ & $0,30 \mathrm{a}$ \\
\hline Omissão de S & $41,04 \mathrm{a}$ & $33,18 \mathrm{a}$ & $7,86 \mathrm{a}$ & $0,33 \mathrm{a}$ \\
\hline $\mathrm{CV}(\%)$ & 54,57 & 56,80 & 54,31 & 44,00 \\
\hline Média & 35,88 & 27,92 & 7,95 & 0,34 \\
\hline Valor de $\mathrm{F}^{(2)}$ & $1,39 \mathrm{NS}$ & 1,36 NS & $1,28 \mathrm{NS}$ & $1,16^{\mathrm{NS}}$ \\
\hline
\end{tabular}

${ }^{(1)}$ Médias seguidas pela mesma letra nas colunas não apresentam diferença significativa, a $5 \%$ de probabilidade pelo teste de Tukey.

(2)***,**,* e NS: significativo ao nível de $1 \%, 5 \%, 10 \%$ de probabilidade e não significativo.

Comportamento semelhante foi observado por Duboc (1994), em mudas de jatobá (Hymenaea courbaril L. var.), onde a produção de matéria seca não variou significativamente.

\section{CONCLUSÕES}

A omissão dos macronutrientes resultou em alterações morfológicas traduzidas em anormalidades visíveis, sendo que, $\mathrm{N}, \mathrm{K}, \mathrm{Ca}$ e $\mathrm{Mg}$ quando faltantes causaram os primeiros sintomas, seguidos pelo $\mathrm{P}$ e $\mathrm{S}$.

Cerne, Lavras, v. 17, n. 3, p. 347-352, jul./set. 2011 
As omissões de $\mathrm{N}, \mathrm{Ca}$ e $\mathrm{Mg}$ foram as que mais influenciaram negativamente o desenvolvimento da espécie em altura, diâmetro e número de folhas e ramos.

A produção de biomassa das mudas foi pouco influenciada pelos tratamentos não apresentando diferença significativa, mas isso deve-se, em parte, ao pequeno número de repetições e elevado coeficiente de variação no experimento.

\section{AGRADECIMENTO}

A Fundação de Amparo à Pesquisa do Estado de São Paulo pela concessão de auxílio pesquisa bolsa de estudos (processo n0213193-7; 0213197-3)

\section{REFERÊNCIAS}

ARAUJO, P. A.; MACHADO, C. T. T. Fósforo. In: FERNANDES, M. S. Nutrição mineral de plantas. Viçosa, MG: Sociedade Brasileira de Ciências do Solo, 2006. p. 253-298.

DUBOC, E. Enriquecimento nutricionais de espécies florestais nativas: Hymenaea courbaril, Copaifera langsdorffii e Peltophorum dubium. 1994. 68 f. Dissertação (Mestrado) - Universidade Federal de Lavras, Lavras, 1994.

DURIGAN, G.; FIGLIOLIA, M. B.; KAWABATA, M.; GARRIDO, M. A. O.; BAITELLO, J. B. Sementes e mudas de árvores tropicais. 2. ed. São Paulo: Instituto Florestal, 2002. 65 p.

EPSTEIN, E.; BLOOM, A. J. Mineral nutrition of plants: principles and perspectives. 2. ed. Sunderland: Sinauer Associates, 2005. 400 p.

JOHNSON, C. M.; STOUT, P. R.; BROYER, T. C.; CARTON, A. B. Comparative chlorine requirements of different plant especies. Plant and Soil, The Hague, v. 8, n. 4, p. 337-353, 1957.

LORENZI, H. Árvores brasileiras: manual de identificação e cultivo de plantas arbóreas nativas do Brasil. Nova Odessa: Instituto Plantarum, 1992. $352 \mathrm{p}$.

LORENZI, H. Plantas medicinais no Brasil: nativas e exóticas cultivadas. Nova Odessa: Instituto Plantarum, 2002. 512 p.

MALAVOLTA, E. Manual de nutrição mineral de plantas. São Paulo: Agronômica Ceres, 2006. 638 p.

Cerne, Lavras, v. 17, n. 3, p. 347-352, jul./set. 2011
MALAVOLTA, E.; CROCOMO, J. O. Potássio e a planta In: _. Potássio na agricultura brasileira. Piracicaba: Instituto da Potassa \& Fosfato, 1982. p. 95-162.

MALAVOLTA, E.; VITTI, G. C.; OLIVEIRA, S. A. Avaliação do estado nutricional das plantas: princípios e aplicações. 2. ed. Piracicaba: Associação Brasileira para a Pesquisa da Potassa e do Fosfato, 1997. 319 p.

MARQUES, T. C. L. L. M.; CARVALHO, J. G. de; LACERDA, M. P. C.; MOTA, P. E. F. da. Exigências nutricionais do paricá (Schizolobium amazonicum, Herb.) na fase de muda. Cerne, Lavras, v. 10, n. 2, p. 167-183, 2004.

MARSCHNER, H. Mineral nutrition of higher plants. 2. ed. London: Academic, 1995. 889 p.

MENDONÇA, A. V. R.; NOGUEIRA, F. D.; VENTURIN, N.; SOUZA, J. S. Exigências nutricionais de Myracrodruon urundeuval Fr. All (aroeira do sertão). Cerne, Lavras, v. 5, n. 2, p. 65-75, 1999.

MENGEL, K.; KIRKBY, E. A. Principles of plant nutrition. Bern: International Potash Institute, 1987. 687 p.

RAIJ, B. van. Fertilidade do solo e adubação. Piracicaba: Agronômica Ceres, 1991. 343 p.

SANGINGA, N.; GWAJE, D.; SWIFT, M. J. Nutrient requeriments of exotic tree species in Zimbabwe. Plant and Soil, Dordrecht, v. 132, p. 197-205, 1991.

SORREANO, M. C. M.; MALAVOLTA, E.; SILVA, D. H.; CABRAL, C. P.; RODRIGUES, R. R. Deficiência de micronutrientes em mudas de sangra d'água (Croton urucurana, Baill.). Cerne, Lavras, v. 14, p. 127-132, 2008.

TAIZ, T.; ZEIGER, E. Fisiologia vegetal. 3. ed. Porto Alegre: Artmed, 2004. 719 p.

VENTURIN, N.; SOUZA, P. A. de; MACEDO, R. L. G. de. Adubação mineral da candeia (Eremanthus erythropappus (DC.) McLeish). Floresta, Curitiba, v. 35, n. 2, p. 211-219, maio/ago. 2005.

VIÉGAS, I. de J. M.; BATISTA, M. M. F.; FRAZÃO, D. A. C.; CARVALHO, J. G. de; SILVA, J. F. da. Avaliação dos teores de N, P, K, Ca, Mg e S em plantas de gravioleira cultivadas em solução nutritiva com omissão de macronutrientes. Revista de Ciências Agrárias, Belém, n. 38, p. 17-28, 2002. 